\title{
Elektronische Geldbörsen in Deutschland
}

von Wolfgang Gentz, Koch Unternehmensberatung, München

Beim Einkauf im Einzelhandel und an Automaten kann Bargeld durch eine sogenannte Elektronische Geldbörse ersetzt werden. Elektronische Geldbörsen sind, wie in vielen anderen Ländern auch, in Deutschland bereits technisch realisiert und in der Anwendung. In Deutschland existieren zur Zeit drei voneinander unabhängige Systeme: die GeldKarte, die PayCard und die P-Card. Alle Systeme basieren auf der Chipkartentechnik. Bei diesen drei Geldbörsensystemen wurden unterschiedliche Ansätze gewählt, die auf die Interessen der unterschiedlichen Initiatoren und Herausgeber der Geldbörsen zurückgeführt werden können. Im folgenden geht es um eine vergleichende Darstellung der Funktionsweise dieser drei Systeme, um erste Erfahrungen aus der Markteinführung und um einige Aspekte ihrer Wirtschaftlichkeit.

\section{Aufbau der Geldbörsensysteme}

Alle genannten Systeme basieren auf der Chipkartentechnik. Jeder Teilnehmer des Systems erhält eine Chipkarte mit einem Mikroprozessor. Chipkarten (mit einer etwas anderen Technologie) sind als Telefonkarte oder als Krankenversicherungskarte bereits weit verbreitet. Chipkarten im Zahlungsverkehr sind dagegen eine neue Variante unter den Zahlungskarten für den bargeldlosen Zahlungsverkehr. Dazu zählen erstens die Kreditkarten, zweitens die Debitkarten (z.B. die Eurocheque-Karte mit dem electronic cash-Verfahren) und drittens die elektronischen Geldbörsen. Diese unterscheiden sich u.a. durch den Zahlungszeitpunkt. Bei den Kreditkarten wird zeitlich nach dem Kaufvorgang die Zahlung abgewickelt ("pay later"), bei den Debitkarten wird das Kundenkonto sofort belastet ("pay now") und bei den Geldbörsen wird ein Guthaben auf die Karte vorausbezahlt ("prepaid").

Folgt man der Europäischen Norm prEN1546 zu elektronischen Geldbörsen, dann wird für jeden Ladevorgang eine Datenverbindung aufgebaut, während die Bezahlungsvor- 
gänge offline erfolgen. Das Konzept geht von Zahlungsvorgängen in geringer Höhe aus. Die Aufladung kann über ein Konto erfolgen, aber auch kontoungebunden. Neben der eigentlichen Geldbörsenfunktion bietet der Chip in der Regel Platz für Zusatzanwendungen wie z.B. elektronische Parkscheine, elektronische Fahrkarten im Nahverkehr oder Bonussysteme im Handel.

Ein Geldbörsensystem erfordert einen hohen technischen und organisatorischen Aufwand. In der Norm prEN1546 werden insgesamt sieben Teilnehmer unterschieden, die jeweils bestimmte Aufgaben übernehmen. Durch Zusammenfassen oder Aufteilen der Aufgaben können sich verschiedene Organisationsstrukturen ergeben. Die drei Hauptbeteiligten sind der Börsenherausgeber, die Börseninhaber und die Akzeptanzstellen, die an die Börseninhaber Waren oder Dienstleistungen verkaufen. Der Börsenherausgeber (in der Regel eine Bank) gibt die Werteinheiten heraus. Er muß für deren Einlösung garantieren, da er den Gegenwert als Vorauszahlung der entsprechenden Geldsumme vom Börseninhaber erhalten hat. Der Börsenanbieter ist für die Sicherheit des Systems und der Chipkarten verantwortlich. Außerdem werden Aufladestellen, Datensammelstellen (zur Sammlung der Daten der Zahlungsvorgänge und Weiterleitung an den Börsenherausgeber), ein Kartenherausgeber und ein Herausgeber für Sicherheitsmodule definiert. Die Rolle einer Verrechnungszentrale wird in der Norm implizit dem Börsenanbieter zugewiesen.

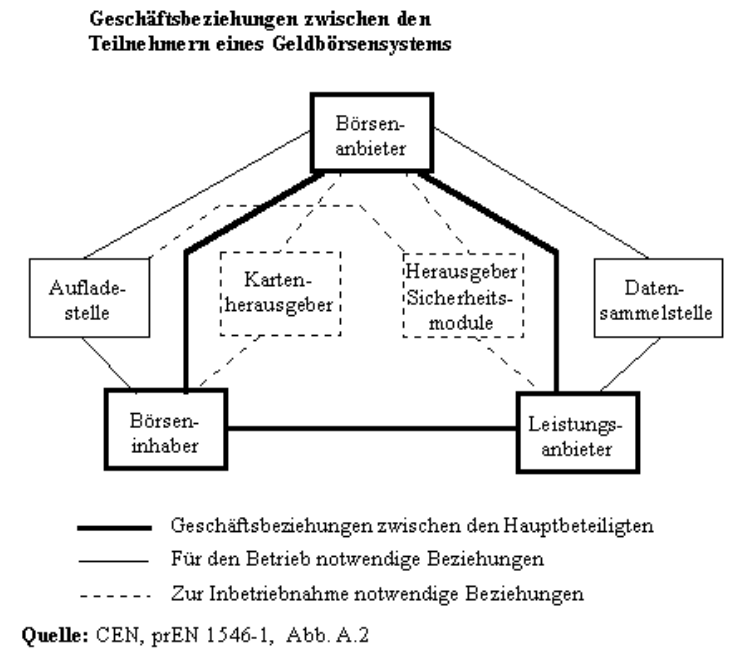

\subsection{GeldKarte}

Die GeldKarte wird von der deutschen Kreditwirtschaft seit Januar 1997 zusammen mit der Eurocheque-Karte bzw. auf Bank-Kundenkarten ausgegeben. Ende 1997 waren schätzungsweise 35 Millionen Geldkarten bei den Kunden, davon wurden allerdings nur wenige auch wirklich aktiv genutzt.

Beim Ladevorgang wird das Konto des Karteninhabers mit dem Ladebetrag belastet (kontogebundene Karten) bzw. das Bargeld hereingenommen (kontoungebundene Karten) und dem Börsenverrechnungskonto der kartenausgebenden Bank gutgeschrieben. Dem kartenausgebenden Institut steht der Geldbetrag solange zur Verfügung, bis die Umsätze wieder belastet werden (Float-Nutzen). Die Börsenevidenzzentrale erhöht den Schattensaldo um den Ladebetrag. Ladeterminals stehen bei den Banken und auch an öffentlichen Plätzen, z.B. in Einkaufszentren, zur Verfügung.

Beim Bezahlvorgang am Händlerterminal wird das Guthaben auf der GeldKarte reduziert und ein eindeutiger Transaktionsdatensatz erstellt, der die Händlerkartennummer, eine fortlaufende Händlertransaktionsnummer sowie eine Lade- und Börsensequenznummer der GeldKarte enthält. Diese Transaktionsdaten werden im Händlerterminal gespeichert und auf der Händlerkarte wird der Saldo um den Betrag der erhaltenen Zahlungen erhöht.

Die Akzeptanzstelle (Händler) führt zur Erstattung der Beträge einen Kassenschluß durch, bei dem die einzelnen im Terminal gespeicherten Transaktionen zu einer Datei zusammengefaßt werden. Die Daten werden i.d.R. per Datenfernübertragung an die Börsenevidenzzentrale eingereicht.

Bei der Börsenevidenzzentrale werden die eingereichten Transaktionen auf gefälschte oder verfälschte Umsätze und Mehrfacheinreichungen überprüft. Die Umsätze werden dem Verrechnungskonto der kartenausgebenden Bank belastet. Der Umsatz abzüglich des sog. Händlerentgelts wird auf das Konto bei der Hausbank des Händlers weitergeleitet. Die Börsenevidenzzentrale berechnet $0,3 \%$ des Umsatzes, mindestens jedoch 0,02 DM pro Transaktion als Händlerentgelt, das wie der Float-Nutzen dem kartenausgebenden Institut zusteht. 
Zusätzlich werden anhand der Einzeltransaktionen die Schattensalden aktualisiert. Mit Hilfe der Schattensalden sollen zum einen Systemangriffe, etwa eine unautorisierte Ladung der Börsenkarte, erkannt werden und zum anderen bei einem Defekt der Börsenkarte eine Erstattung des Restguthabens möglich sein. Der Bundesbeauftragte für den Datenschutz hat sich bereits mehrfach kritisch zu diesen Schattensalden geäußert.

\subsection{PayCard}

Die PayCard ist eine Gemeinschaftsentwicklung der Deutschen Telekom, der Deutschen Bahn und des Verbands Deutscher Verkehrsunternehmen (VDV). Einige Zehntausend PayCards sollen im Markt sein. Sie wird seit März 1997 von der Deutschen Telekom vertrieben, seit März '98 standardmäßig auf der T-Card.

Wie bei der GeldKarte wird zwischen kontogebundenen und kontoungebundenen Börsenkarten unterschieden. Die kontoungebundenen Karten werden gegen Barzahlung aufgeladen. Die Aufladung der kontogebundenen Karten kann an einem der ca. 93.000 Kartentelefone der Deutschen Telekom durchgeführt werden. Das Hintergrundsystem veranlaßt anschließend (per Lastschrift) eine Belastung des Kontos bei der Bank des Kunden.

Die PayCard unterscheidet sich bei der Verrechnung wesentlich von der GeldKarte und der P-Card: Akzeptanzstellen mit hohen Gesamtumsätzen (z.B. Verkehrsbetriebe) können am sog. Subbörsen-Clearing teilnehmen. Die auf Grund der Ladevorgänge eingezogenen Geldbeträge verbleiben so nicht beim Börsenherausgeber, sondern werden durch eine Vorabverteilung zu $75 \%$ an die Akzeptanzstellen (in diesem Fall die Verkehrsbetriebe) weitergegeben, $25 \%$ verbleiben im Pool des Kartenherausgebers.

Ein weiteres wichtiges Merkmal, das die PayCard von der GeldKarte unterscheidet, ist die Speicherung der Transaktionsdaten. Für die Teilnehmer des Subbörsen-Clearings werden die Umsätze nicht in den Zahlungsterminals (hier in der Regel ein Fahrkartenautomat) gespeichert, sondern in den Börsenkarten des Kunden. Beim Bezug von Leistungen wird das Guthaben der Börse des Karteninhabers reduziert und (falls nicht bereits vorhanden) für den
Zahlungsempfänger eine Subbörse angelegt. Dieser Subbörse wird der Betrag gutgeschrieben. Für den Verbraucher ergibt sich aus Sicht des Datenschutzes der Vorteil, daß seine Umsatzdaten nicht in einem Hintergrundsystem gespeichert werden.

Die Teilnehmer des Subbörsen-Clearings müssen keine Umsätze an die Clearingstelle einreichen. Die Verrechnung wird beim nächsten Ladevorgang am Kartentelefon angestoBen. Dazu werden die Summen der einzelnen Subbörsen ausgelesen und an das Hintergrundsystem übertragen. Vom Umsatz wird den Akzeptanzstellen ein Disagio oder eine Transaktionsgebühr in Höhe von 0,3\% abgezogen; eine Mindestgebühr pro Transaktion wird nicht berechnet.

\subsection{P-Card}

Die P-Card wurde von einem Verbund mehrerer Unternehmen (Elektronic Banking Systems, Orga KartenSysteme, Krone Kommunikationstechnik und Göppinger Datensysteme) entwikkelt und gilt als ein händlerorientiertes System. Das System kam bisher in einem 16-monatigen Feldversuch, der Ende Januar 1998 abgeschlossen wurde, in der Stadt Höxter zum Einsatz.

Das Konzept sieht vor, daß der Händler nicht nur als Akzeptant auftreten kann, sondern auch als Kartenherausgeber und zusätzlich als Ladestelle. Neben der Bezahlungsfunktion wird besonderes Augenmerk auf eine Nutzung als Kundenkarte gelegt. Zur Verrechnung werden die Umsätze an die Clearingzentrale eingereicht. Es werden transaktionsabhängige $\mathrm{Ge}$ bühren berechnet, deren Höhe zum einen von der Branche und zum anderen von der Gesamtzahl der Transaktionen abhängt, nicht aber wie bei den anderen Systemen von der Höhe des Umsatzes. Ein weiteres Unterscheidungsmerkmal der P-Card im Vergleich zu den beiden anderen Systemen ist, daß eine Verzinsung der Börsenguthaben für den Kunden vorgesehen ist.

\subsection{Vergleich der drei Geldbörsen in Deutschland}

Die unterschiedlichen Konzepte sind zum Teil auf den Geschäftszweck der Herausgeber der Elektronischen Geldbörsen zurückzuführen. Die GeldKarte der Deutschen Kreditwirtschaft soll 
die Bemühungen unterstützen, den Zahlungsverkehr als Geschäftsfeld der Kreditinstitute gegenüber möglicher Konkurrenz aus dem Ausland bzw. von Nicht-Banken zu sichern und das bestehende electronic cash-System zu ergänzen bzw. dessen Infrastruktur besser auszulasten. Die PayCard als eine von den Verkehrsunternehmen und der Telekom initiierte Karte ist zumindest in der Anfangsphase hauptsächlich im Bereich der Fahrkartenautomaten einsetzbar und soll zur Kostenreduzierung bei den Verkehrsunternehmen beitragen. Sie ist vor allem auch unter Gesichtspunkten des Datenschutzes ein interessantes Konzept.

Die P-Card erfährt Unterstützung aus dem Handel und berücksichtigt insbesondere den Aspekt der Kundenbindung. In der folgenden Tabelle werden nochmals einige wesentliche Merkmale der drei Geldbörsen gegenübergestellt.

\begin{tabular}{|c|c|c|c|}
\hline & GeldKarte & PayCard & P-Card \\
\hline Ladestellen & $\begin{array}{l}19.700(12 / 97) \text { Ladeterminals } \\
\text { insbesondere bei Kreditinstitu- } \\
\text { ten }\end{array}$ & 93.000 Kartentelefone & Ladeterminal beim Händler \\
\hline Höchstsaldo & $400 \mathrm{DM}$ & $400 \mathrm{DM}$ & $400 \mathrm{DM}$ \\
\hline Ladegebühr & $\begin{array}{l}\text { Bei eigener Bankgruppe keine, } \\
\text { bei fremder Bank } 2 \text { DM }\end{array}$ & 0,60 DM pro Ladevorgang & 0,80 DM pro Ladevorgang \\
\hline Akzeptanzstellen & $\begin{array}{l}50.400(12 / 97) \text { Terminals } \\
\text { insbesondere im Einzelhan- } \\
\text { del }\end{array}$ & $\begin{array}{l}\text { einige Hundert Fahr- } \\
\text { scheinautomaten }(11 / 97), \\
\text { Nutzung als Telefonkarte }\end{array}$ & $\begin{array}{l}\text { vorgesehen im Einzelhandel } \\
\text { und bei Automaten }\end{array}$ \\
\hline $\begin{array}{l}\text { Transaktionskosten } \\
\text { für Akzeptanzstelle }\end{array}$ & $0,3 \%$, mindestens $2 \mathrm{Pf}$. & $0,3 \%$ & $\begin{array}{l}\text { transaktionsbezogen zwi- } \\
\text { schen } 3,5 \text { und } 35 \mathrm{Pf} \text {. }\end{array}$ \\
\hline $\begin{array}{l}\text { Speicherung der } \\
\text { Verkaufsdaten }\end{array}$ & im Händlerterminal & auf der PayCard & im Händlerterminal \\
\hline Markteinführung & $\begin{array}{l}\text { seit Januar } 1997 \text { auf der } \\
\text { Eurocheque-Karte bzw. } \\
\text { Kundenkarte }\end{array}$ & seit März 1997 & noch nicht erfolgt \\
\hline Karten im Markt & $\begin{array}{l}\text { etwa } 35 \text { Mio. Ende } 1997 \text {, } \\
\text { davon } 480.000 \text { aktiv }\end{array}$ & einige Zehntausend & noch keine Markteinführung \\
\hline Umsatz & 87 Mio. DM in 1997 & $86.000 \mathrm{DM}$ von $5-8 / 97$ & $\begin{array}{l}\text { 320.000 DM (während des } \\
\text { 16-monatigen Pilotversuchs } \\
\text { in Höxter) }\end{array}$ \\
\hline $\begin{array}{l}\text { Durchschnittlicher } \\
\text { Bezahlvorgang }\end{array}$ & 20,78 DM (1997) & nicht bekannt & $\begin{array}{l}\text { 23,87 DM (während Pilot- } \\
\text { versuch Höxter) }\end{array}$ \\
\hline
\end{tabular}

Tabelle: Vergleich der drei deutschen Geldbörsensysteme

\section{Wirtschaftlichkeit der Geldbörsensy- steme am Beispiel GeldKarte ${ }^{1}$}

Für das Jahr 1997 liegt eine von der Kreditwirtschaft (ZKA, Zentraler Kreditausschuß) erstellte Statistik vor:

- Die Zahl der aktiven Chipkarten stieg vom Januar '97 von ca. 78.000 auf ca. 480.000 im Dezember '97 (im Bezugsmonat mindestens einmal aufgeladen oder mindestens einmal bezahlt).
- 1997 wurden insgesamt ca. 209 Mio. DM auf die GeldKarten aufgeladen. Die Summe der Bezahltransaktionen lag bei ca. 87 Mio. DM, die Entladetransaktionen (also die Auszahlungen von GeldKarten-Guthaben an den GeldKarten-Inhaber oder auf dessen Konto) beliefen sich auf ca. 25 Mio. DM. Die verhältnismäßig hohe Summe der Entladungen könnte sich mit den relativ wenigen Akzeptanzstellen erklären lassen. 
- Durchschnittlich wurden je Ladetransaktion ca. 134,-- DM aufgeladen. Der durchschnittliche Bezahlbetrag lag bei 20,78 DM.

Für den Herausgeber der Börsenkarten stehen zwei wichtige Einnahmequellen zur Verfügung: Der Ertrag aus dem Disagio, das dem Händler für die Transaktionskosten berechnet wird und die Nutzung des Betrages, der als Differenz zwischen den Ladebeträgen und den Bezahlund Entladevorgängen zur Verfügung steht (Float-Nutzen).

Legt man die Umsatzzahlen von 87 Mio. DM für $1997 \mathrm{zu}$ Grunde, so ergibt sich daraus, ohne Berücksichtigung von Mindestgebühren, ein Gebührenertrag von 261.000 DM (0,3\%), unter Hinzurechnung der Mindestgebühren für Umsätze unter 6,67 DM liegt der Betrag etwas höher. Unter Berücksichtigung der Schwankungen läßt sich der Betrag, der durchschnittlich auf den Börsenverrechnungskonten der Kartenherausgeber zur Verfügung stand, auf ca. 37 Mio. DM schätzen. Jeder Prozentpunkt, mit dem sich dieses Kapital am Geldmarkt anlegen läßt, entspricht einem jährlichen Zinsertrag von 370.000 DM, bei Zugrundelegung eines Geldmarktsatzes unter Banken in Höhe von 3,5\% (Tagesgeld) entspricht dies ca. 1,3 Mio. DM für das Gesamtsystem. Daraus wird ersichtlich, daß die Einnahmen aus dem Float-Nutzen deutlich höher liegen als die Gebühreneinnahmen durch den Einsatz der GeldKarte. In Modellrechnungen konnte gezeigt werden, daß dies auch bei einer Erhöhung der aktiven Karten und einer Erhöhung des Umsatzes pro Karte so bleibt. Neben diesen Erträgen spielen auch Kosteneinsparungen der Kreditinstitute durch vermindertes Bargeld-Handling und die verstärkte Nutzung bestehender Zahlungssysteme eine Rolle.

Den Erträgen stehen die Kosten gegenüber: Herstellung der Börsenkarten, Kosten für Ladegeräte und Aufbau des Hintergrundsystems sowie der Marketingaufwand. Für den Verrechnungsaufwand der Börsenevidenzzentralen werden die kartenausgebenden Institute mit Kosten belastet, die sicherlich über den derzeit erzielten Gebührenerträgen liegen. Zum jetzigen Zeitpunkt ist davon auszugehen, daß das GeldKarten-System noch weit von einer Kostendeckung entfernt ist und diese auch in absehbarer Zeit nicht zu erwarten ist. In Mo- dellrechnungen konnte gezeigt werden, daß dies in gleicher Weise auch für die PayCard gilt.

\section{Ausblick}

Der elektronische Handel im Internet mit der Möglichkeit der sicheren Bezahlung gewinnt an Aktualität. Auf der CeBIT '98 wurden mehrere Realisierungen vorgestellt, die auch eine Nutzung der GeldKarte bzw. P-Card für den sicheren Bezahlvorgang im Internet ermöglichen. Sollte sich diese Anwendung etablieren, würde dies die allgemeine Nutzung von elektronischen Geldbörsen im Handel und an Automaten ebenfalls fördern.

Für den Verbraucher ergibt sich allerdings das Problem, daß er mehrere Börsenkarten besitzen muß, um sowohl im Einzelhandel, im ÖPNV, an öffentlichen Kartentelefonen und eventuell im Internet die Elektronische Geldbörse einsetzen zu können. Ein hemmender Faktor für die Akzeptanz der deutschen Geldbörsensysteme ist darüber hinaus, $\mathrm{da} \beta$ sie in Konkurrenz stehen zu einer Vielzahl konkurrierender europäischer Systeme, was sich insbesondere nach der Einführung einer einheitlichen europäischen Währung als Problem herausstellen kann.

Konkurrenz erwächst den Geldbörsen möglicherweise auch durch die Kreditkarten. In Deutschland waren im Januar 1996 über 11,75 Millionen Kreditkarten ausgegeben, das Potential wird auf 20 Millionen Stück geschätzt. Die Kreditkartenunternehmen verfügen über ein weltweites Akzeptanzstellennetz, das für den Einsatz einer Elektronischen Geldbörse genutzt werden kann. $\mathrm{Zu}$ beachten ist allerdings, daß es sich bei rund $85 \%$ der ausgegebenen Kreditkarten um sog. Bankkreditkarten handelt, also um von Kreditinstituten in Lizenz emittierte Karten. Wie viele dieser Kreditkarten als Träger für den Chip einer elektronischen Geldbörse dienen werden, hängt von der Geschäftspolitik der ausgebenden Banken oder aber von den zukünftigen Lizenzbedingungen ab.

\section{Anmerkung}

1 Eine umfassende Wirtschaftlichkeitsbetrachtung der elektronischen Geldbörsensysteme ist in Gentz 1997 vorgenommen worden. Im Rahmen dieses Artikels können hier nur einige Hinweise gegeben werden. 


\section{Literatur}

Gentz, Wolfgang: Die elektronische Geldbörse in Deutschland: Funktionsweise, Kosten und Nutzen für die Beteiligten, Diplomarbeit an der Fachhochschule München, 1997; http://www.ubkoch.de/diplom.html.

Hamburg-Consult, Begleituntersuchung zur Einführung der PayCard im ÖPNV - Schlußbericht zum FE-Projekt Nummer 70482/96, Hamburg, 1997.

Kubicek, Herbert/Klein, Stephan: Wertkarten im Zahlungsverkehr - Trends und Perspektiven auf dem Weg zur elektronischen Geldbörse, Wiesbaden: Gabler, 1995.

Rankl, Wolfgang/Effing Wolfgang: Handbuch der Chipkarten - Aufbau - Funktionsweise - Einsatz von Smart Cards, 2. überarb. u. erw. Aufl., München/Wien: Hanser, 1996.

TransTec Transport und Technologie Consult Hannover: Akzeptanzuntersuchung der GeldKarte in Ravensburg/Weingarten und Hannover - Schlußbericht, Forschungsvorhaben im Auftrag des Bundesministeriums für Verkehr, 1997.

\section{Kontakt}

Wolfgang Gentz

Koch Unternehmensberatung $\mathrm{GmbH}$

Ottostr. 5, D-80333 München

Tel.: + 49 (0) 89/545932-17

E-mail: Wolfgang.Gentz@munich.netsurf.de 